http://www.jrms.dentaiau.ac.ir
JRDMS Journal of Research in Dental and Maxillofacial Sciences

\title{
The Correlation between Cigarette Smoking and Salivary Flow Rate
}

\author{
Alaee A $\mathbf{A}^{1}$,Azizi A ${ }^{2}$, Valaei N², Moeini SH ${ }^{*}$ \\ ${ }^{l}$ Assistant Professor, Oral medicine Dept, Member of Dental Material Research Center, Dental Branch of Tehran, Islamic Azad University, Tehran,Iran. \\ ${ }^{2}$ Professor,Oral medicine Dept, Dental Branch of Tehran, Islamic Azad University, Tehran,Iran. \\ ${ }^{3}$ Faculty member of Thalasemia Research center, Mazandaran ,Iran \\ ${ }^{4}$ Dentist
}

\begin{tabular}{l}
\hline ARTICLE INFO \\
\hline Article Type \\
Orginal Article \\
\hline Article History \\
Received: Apr 2017 \\
Accepted: May 2017 \\
ePublished: June 2017
\end{tabular}

Keywords:

Smoking,

Cigarette,

Mouth Dryness,

Saliva

\begin{abstract}
Background and aim: Side effects of cigarette smoking are among the major concerns. These complications can adversely affect the oral environment. Since reduced salivary flow rate increases the incidence of tooth decay and other dental and oral problems, the present research aimed to investigate the relationship between cigarette smoking and salivary flow rate.

Materials and Methods: In this historical cohort study, 50 cigarette smokers (case) and 50 non-smokers (control) were involved, who were matched according to age and gender. Non-stimulated whole saliva was measured by using the modified Schirmer test (MST) performed between 9 a.m. and 12 p.m. by a trained examiner. All the participants refrained from eating, drinking and smoking for 2 hours prior to the study. The subjects were asked to sit in an upright position and to raise and slowly retract their tongue, to avoid unintentional wetting of the Schirmer test's strip. The strip was kept vertically with the help of cotton pliers, while the bottom of the paper was in contact with the floor of the mouth. The length of the wet area on the strip was recorded at the intervals of 1, 2 and 3 minutes. Data were analyzed with Mann-U-Whitney and Chi-square tests.

Results: The quantitative value of salivary flow rate was equal to $24.8 \pm 2.4 \mathrm{~mm}$ in controls, and $15.8 \pm 2.1 \mathrm{~mm}$ in the case group $(\mathrm{P}<0.001) .30 \%$ of non-smokers and $90 \%$ of smokers exhibited reduced salivary flow rate $(\mathrm{P}<0.000)$.
\end{abstract}

Conclusion: It seems that reduced salivary flow rate is more significant in cigarette smokers than in non-smokers. 


\section{Introduction:}

Xerostomia, a condition in which saliva is secreted in reduced amounts, is an annoying condition that may have a life-long duration. It can cause complications such as dysphagia, increased risk of candida infection, dysgeusia, impaired nutritional status, tongue papillae atrophy, lobulated tongue, halitosis and cervical tooth decay. ${ }^{(1-3)}$

Overall, oral and dental problems can negatively affect the patient's quality of life by compromising his/her physical performance, social performance and self-confidence. ${ }^{(1,2)}$ The most common causes of xerostomia are salivary gland atrophy, usage of certain drugs, head and neck radiotherapy, Sjögren's syndrome and systemic diseases. ${ }^{(2)}$

At present, smoking is one of the most important risk factors for developing oral lesions, tooth discoloration, halitosis, hairy tongue, increased calculi, periodontal diseases, mouth dryness, and oropharyngeal and respiratory cancers. ${ }^{(4,5)}$

In the absence of patient's awareness of tobacco side effects and with continuous tobacco consumption, carcinogenic metabolites of tobacco react with DNA and may initiate carcinogenesis in the oral mucosa. ${ }^{(5,6)}$

There are conflicting reports about the correlation between cigarette smoking and mouth dryness, as some studies have mentioned that cigarette smokers have lower salivary flow rate than non-smokers, ${ }^{(5)}$ whereas other studies have shown that cigarette smoking has no effect on mouth dryness. ${ }^{(7,8)}$

Considering the controversies and given the fact that to date no other research has explored the association between cigarette smoking and xerostomia in Iran, the aim of this study was to investigate the correlation between cigarette smoking and mouth dryness, according to the modified Schirmer test.

\section{Materials and Methods:}

The protocol of this historical cohort study was approved by the ethics committee of the dental branch of Islamic Azad University of Tehran. 50 cigarette smokers were placed in the case group after consenting to the study plan. The con- trol group consisted of 50 non-smokers.

The two groups were matched according to age and gender. The participants were not consuming any medications, had no systemic diseases and were not using any other tobacco products. Each of the subjects was given an Oral-B toothpaste and toothbrush by the examiner to raise their level of cooperation.

Non-stimulated whole saliva was measured by using the modified Schirmer test (MST). According to the study by Fontana and colleagues, the MST was performed for all participants between 9 a.m. and 12 p.m. by an examiner trained by an oral medicine specialist. ${ }^{(5)}$

All the participants refrained from eating, drinking and smoking for 2 hours prior to the study. The subjects were asked to sit in an upright position on the dental chair.

The participants were instructed to swallow once to clear the mouth, and not to swallow during the test. Also, they were asked to raise and slowly retract their tongue, to avoid unintentional wetting of the Schirmer test's strip.

The strip was kept vertically with the help of cotton pliers, while the bottom of the paper was in contact with the floor of the mouth, both on the right and left sides of the lingual frenulum (Fig. $1) .^{(5)}$

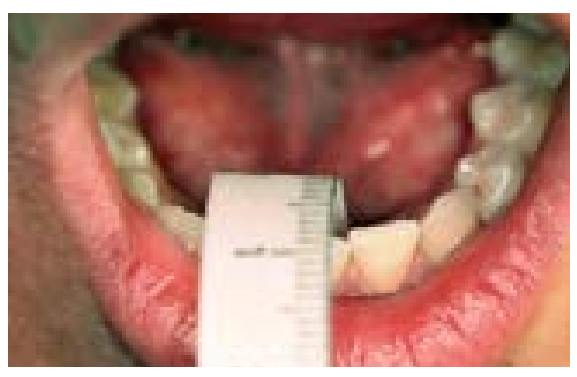

Figure 1: Measurement of salivary flow rate by the modified Schirmer test.

The color of the strip changes to brown in contact with moisture. The length of this brown area on the strip was recorded by the examiner at the intervals of 1,2 and 3 minutes. The mean length of the wet areas on the two strips was calculated. In the present study, less than $25 \mathrm{~mm}$ of moisture during 3 minutes was considered reduced salivary flow rate. ${ }^{(5)}$ 
Table 1- Characteristics of the study population according to cigarette smoking status.

\begin{tabular}{lccccccc}
\hline & \multicolumn{2}{c}{ Sex } & & \multicolumn{3}{c}{ Education level } \\
\cline { 2 - 3 } \cline { 7 - 8 } Cigarette smoking & Male & Female & \multirow{2}{*}{$\begin{array}{c}\text { Age } \\
\text { (year) }\end{array}$} & College & $\begin{array}{c}\text { High } \\
\text { school }\end{array}$ & $\begin{array}{c}\text { Middle } \\
\text { school and } \\
\text { lower }\end{array}$ \\
\hline No (Control) $\mathrm{n}=50$ & 26 & 24 & $43 \pm 8.2$ & 21 & 29 & $(-) 0$ \\
\hline Yes (Case) $\mathrm{n}=50$ & 32 & 18 & $41 \pm 3.2$ & 18 & 32 & $(-) 0$ \\
\hline
\end{tabular}

\section{Results:}

This study was conducted on 100 individuals, including 50 non-smokers (controls) and 50 cigarette smokers (test group).

All participants were identical with regards to simultaneous reference to the dental school and therefore, were similar in terms of economic and social status.

The characteristics of the study population categorized by cigarette smoking status are presented in Table 1, which shows that the two groups were similar in terms of gender, age and education level $(\mathrm{P}>0.2)$.

The quantitative and qualitative amounts of saliva categorized by cigarette smoking status are presented in Table 2, which shows that the quantitative amount of saliva in the control group was equal to $24.8 \pm 2.4 \mathrm{~mm}$, while in the group of cigarette smokers it was equal to $15.8 \pm 2.1 \mathrm{~mm}$, which was $9 \mathrm{~mm}$ less or $36.3 \%$ lower in smokers. MannU-Whitney test showed that this difference is statistically significant $(\mathrm{P}<0.001)$.

Table 2- Quantity and quality of saliva according to cigarette smoking status.

\begin{tabular}{lccc}
\hline \multirow{2}{*}{$\begin{array}{c}\text { Cigarette } \\
\text { smoking }\end{array}$} & $\begin{array}{c}\text { Amount } \\
(\mathbf{m m})\end{array}$ & \multicolumn{2}{c}{ Reduced saliva } \\
\cline { 3 - 4 } & & No & YES \\
\hline No (Control) $\mathrm{n}=50$ & $24.8 \pm 2.4$ & $35(70 \%)$ & $15(30 \%)$ \\
\hline Yes $($ Case $) \mathrm{n}=50$ & $15.8 \pm 2.1$ & $5(10 \%)$ & $45(90 \%)$ \\
\hline Test result & $\mathrm{P}<0.001$ & \multicolumn{2}{c}{$\mathrm{P}<0.000$} \\
\hline
\end{tabular}

Qualitatively, 30\% of non-smokers and $90 \%$ of cigarette smokers showed reduced salivary flow rate, and Chi-square test showed that this difference is statistically significant $(\mathrm{P}<0.000)$.

\section{Discussion:}

The present study showed that cigarette smoking causes a more significant reduction in salivary flow rate in comparison with non-smoking status. It should be noted that the heat produced during cigarette smoking gradually affects the blood flow and reduces the blood supply, and subsequently decreases saliva. Immunoglobulins (especially IgA) and other protective agents in the blood also decrease due to smoking. The toxins present in cigarettes gradually affect the salivary parenchyma and destruct the salivary glands. ${ }^{(3,5)}$ Carbon monoxide is one of the main toxic gases in cigarettes, which reduces the salivary flow through vitamin A breakdown and restriction of the blood flow. ${ }^{(3,5,6)}$

In a case-control study, Dyasanoor and Saddue used the MST to investigate the correlation between mouth dryness and salivary flow in 60 smokers and 60 non-smokers. They showed that xerostomia symptoms were significantly associated with reduced non-stimulated salivary flow in long-term cigarette smokers $(\mathrm{P}=0.003){ }^{(5)}$ The results of the cited study are consistent with the findings of the current study. Perhaps, cigarette smoking has a greater effect on non-stimulated salivary flow than on stimulated salivary flow.

Rad et al investigated the long-term effects of cigarette smoking on salivary flow rate and oral health. Their case-control study involved 100 people. They concluded that long-term cigarette smoking significantly reduces the salivary flow rate and increases oral and dental disorders associated with xerostomia, particularly cervical caries, gingivitis, loosened teeth, halitosis and dental calculi. ${ }^{(9)}$ These results are also in agreement with the findings of the present study. Perhaps, longterm cigarette smoking has a greater influence on the salivary flow rate..$^{(9-1)}$ 
Ahmadi-Motamayel et al investigated the prevalence of oral mucosal lesions in male cigarette smokers and non-smokers. Their results showed that the most common lesions among the smokers were tongue and gingival lesions, and smokers had significantly more lesions than non-smokers. Malignant and pre-malignant lesions were observed in older age. They found a large number of oral mucosal lesions in smokers, which had a strong association with cigarette smoking. ${ }^{(12)}$

The results of the mentioned study are in line with the findings of the present research. Perhaps, the higher prevalence of oral mucosal lesions among cigarette smokers is due to the impact of smoking on the amount of saliva.

Patil et al conducted a cross-sectional study to assess the prevalence of oral lesions in dental patients with the habit of tobacco smoking, tobacco chewing or both. The results of their study showed that the risk of the progression of oral lesions associated with tobacco smoking, tobacco chewing or both, is high. The men who had one or more of these habits showed more oral changes than women. ${ }^{(13)}$

Nevertheless, the results of the present study are consistent with previous studies indicating that cigarette smoking affects the salivary flow rate. This may be due to the presence of harmful carcinogenic agents in cigarettes, which affect the salivary flow rate and make smokers more susceptible to oral mucosal diseases. ${ }^{(5,9,13)}$

In a case-control study, Khan et al assessed the effects of cigarette smoking on salivary flow rate. They rubbed raw nicotine and citric acid solutions on the tip of the tongue of the subjects. All saliva samples were collected under resting conditions. Their results showed that long-term cigarette smoking does not have any side effects on the response of the taste receptors and therefore has no effect on salivary flow rate. ${ }^{(10)}$

The results of the mentioned study are inconsistent with the findings of our study. This difference can be attributed to different study methods. Furthermore, the amount of nicotine absorbed through the tongue may vary in different individuals, and may render unreliable results.
Conclusion: It seems that reduced salivary flow rate is more significant in cigarette smokers than in non-smokers.

\section{Acknowledgement:}

Our appreciation is extended to all the participants of this study. We are also thankful to the supervisors and advisors of the dental branch of Tehran, Islamic Azad University for the cooperation during this research project which is based on general dentistry thesis, No:25169.

\section{References:}

1.Meyer-Lueckel H, Schulte-Mönting J, Kielbassa AM. The effect of commercially available saliva substitutes on predemineralized bovine dentin in vitro. Oral Dis 2002;8(4):192-8.

2.Sahba S, Ghadimi S, Talaeepour AR, Haddad P, Zolfeghari I. The Efficacy of Iranian Made Saliva Substitute vs. VA-OraLube in Improvement of OralHealth-Related Quality of Life in Radiotherapy-Induced Xerostomia. J Dent Sch 2009;27(3):136-45.

3.Porter SR, Scully C, Hegarty AM. An update of the etiology and management of xerostomia. Oral Surg Oral Med Oral Patho Oral Radio Endod 2004;97(1):28-46.

4.Davis JM. Tobacco cessation for the dental team: A practical guide part 1: Background and Overview. J Contemp Dent Pract 2005;6(3):158-66.

5.Dyasanoor S, Saddu SC. Association of Xerostomia and Assessment of Salivary Flow Using Modified Schirmer Test among Smokers and Healthy Individuals: A Preliminutesary Study. J Clin Diagn Res 2014;8(1):211-3.

6.Warnakulasuriya S, Sutherland G, Scully C. Tobacco, Oral cancer, and treatment of dependence. Oral Oncol 2005;41(3):244-60.

7.van der Putten GJ, Brand HS, Schols JM, de Baat C. The diagnostic suitability of a xerostomia questionnaire and the association between xerostomia, hyposalivation and medication use in a group of nursing home residents. Clin Oral Investig 2011;15(2):185-92. 8.Crews KM, Gordy FM, Penton-Eklund N, Curran AE, Clay JR. Tobacco Cessation: a practical dental service. Gen Dent 1999;47(5):476-83.

9.Rad M, Kakoie S, Niliye Brojeni F, Pourdamghan N. Effect of Long-term Smoking on Whole-mouth Salivary Flow Rate and Oral Health. J Dent Res Dent Clin Dent Prospects 2010;4(4):110-4.

10.Khan GJ, Javed M, Ishaq M. Effect of smoking on salivary flow rate. Gomal J Med Sci 2010;8(2):221-4. 11.Nozad-Mojaver Y, Mirzaee M, Jafarzadeh A. Synergistic effects of cigarette smoke and saliva. Med Oral Patol Oral Cir Bucal 2009;14(5):E217-21. 
12.Ahmadi-Motamayel F, Falsafi P, Hayati Z, Rezaei F, Poorolajal J. Prevalence of Oral Mucosal Lesions in Male Smokers and Nonsmokers. Chonnam Med J 2013;49(2):65-8.

13.Patil PB, Bathi R, Chaudhari S. Prevalence of oral mucosal lesions in dental patients with tobacco smoking, chewing, and mixed habits: A cross-sectional study in South India. J Family Community Med $2013 ; 20(2): 130-5$. 